\title{
Frequência de Adenocarcinoma de Endométrio em Ambulatório de Histeroscopia: Um Estudo Multicêntrico
}

\author{
Endometrial Adenocarcinoma Frequency in a Hysteroscopy \\ Outpatient Clinic: A Multicenter Study
}

\begin{abstract}
Francesco Antonio Viscomi, Sonia Maria Rolim Rosa Lima, José Mendes Aldrighi, Mauro Fernando Kürten Ihlenfeld
\end{abstract}

\begin{abstract}
RESUMO
Objetivo: realizar um censo sobre a freqüência do adenocarcinoma de endométrio em mulheres submetidas a histeroscopia diagnóstica em cinco serviços brasileiros de vídeoendoscopia ginecológica localizados em São Paulo, Rio de Janeiro, Salvador, Caxias do Sul e Porto Alegre.

Métodos: foram utilizados questionários padronizados com o objetivo de obter as informações sobre a presença de adenocarcinoma, acometimento da cavidade uterina e tipo histológico nos exames histeroscópicos de mulheres na pré e pós-menopausa.

Resultados: entre os 6.466 procedimentos histeroscópicos realizados, foi diagnosticado adenocarcinoma de endométrio em 92 pacientes (1,4\%), sendo este diagnóstico confirmado pela histologia em 79 casos (1,2\%). Para o diagnóstico histeroscópico de adenocarcinoma de endométrio, confirmado por exame histológico, obtivemos sensibilidade de 85,9\%, especificidade de $100 \%$, valor preditivo positivo de $100 \%$ e valor preditivo negativo de $98,6 \%$. No grupo de 3.845 pacientes na pré-menopausa, o carcinoma foi diagnosticado pela histeroscopia em $9(0,2 \%)$ casos e confirmados pela histologia em $8(0,2 \%)$, ao passo que no outro grupo de 2.621 pacientes na pós-menopausa, a histeroscopia diagnosticou adenocarcinoma em 83 (3,2\%) pacientes, dos quais 71 casos (2,7\%) foram confirmados pela histologia.

Conclusões: este estudo alerta para uma maior preocupação em relação ao câncer de endométrio, especialmente na pós-menopausa, revelando a necessidade de haver mais estudos epidemiológicos para que se possam elaborar programas de prevenção e diagnóstico precoce do adenocarcinoma de endométrio.
\end{abstract}

PALAVRAS-CHAVE: Histeroscopia. Endométrio: câncer. Câncer: rastreamento.

\section{Introdução}

Nos Estados Unidos da América, o câncer de endométrio é a quarta neoplasia mais freqüente entre as mulheres, com uma estimativa de 34.000 novos casos diagnosticados e 6 mil

ASMUC - Ambulatório de Saúde da Mulher no Climatério Departamento de Saúde Materno-Infantil

Faculdade de Saúde Pública da Universidade de São Paulo - USP

Correspondência:

Francesco Antonio Viscomi

Avenida Eusébio Matoso, 196

05423-000 - São Paulo - SP óbitos no ano de $1996^{1}$, com uma incidência anual, variando de acordo com a idade, entre 12/ 100.000 mulheres até os 40 anos e 100/100.000 mulheres aos $60 \operatorname{anos}^{2}$. A incidência em mulheres com mais de 45 anos vem aumentando nos últimos anos. Naquele país, o número de novos casos de carcinoma de endométrio era superior a três vezes ao de câncer cervical em 1990 e espera-se uma incidência maior para as próximas décadas em função do aumento da expectativa de vida ${ }^{3}$.

No Brasil, são esperados anualmente 5.685 casos novos de adenocarcinoma de endométrio, com uma taxa de 7,6 casos por 100.000 mulheres, 
variando de 2,0/100.000 na Região Norte a 9,9/ 100.000 na Região Sudeste ${ }^{4}$.

Os fatores de risco para o carcinoma de endométrio incluem terapias estrogênicas prolongadas sem oposição por progestógenos, sindrome dos ovários policísticos, tumores produtores de estrogênios, nuliparidade, ciclos menstruais irregulares, idade precoce de menarca, idade tardia de menopausa, obesidade, diabete melito e hipertensão arterial ${ }^{5}$. Em relação ao uso prolongado de estrogênios sem oposição por progestógenos, há um aumento de 4 a 15 vezes no risco de desenvolvimento de câncer de endométrio ${ }^{6}$. Em geral, as mulheres que usam estrogênios costumam ser mais jovens, brancas e não obesas, e habitualmente têm tumores mais diferenciados, menor invasão miometrial e são diagnosticados em estádios iniciais ${ }^{7}$. No entanto, mulheres não usuárias de estrogenioterapia parecem apresentar mais riscos constitucionais, incluindo baixa paridade, menopausa tardia, obesidade, hipertensão e diabete ${ }^{7}$.

Postula-se a existência de dois tipos patogenéticos de carcinoma endometrial ${ }^{8}$. O primeiro é bem diferenciado, mais comum, representa dois terços dos casos e responde à terapia progestacional com bom prognóstico. O segundo costuma ser indiferenciado, mais invasivo, não responde à terapia progestogênica e apresenta prognóstico desfavorável ${ }^{8}$.

A presença de pólipo e hiperplasia é mais comum em pacientes com carcinoma de endométrio do que em grupos controle ${ }^{9}$. Embora seja dificil analisar a evolução de um quadro de hiperplasia para adenocarcinoma, os estudos de Kurman et al. ${ }^{9}$ indicam que $23 \%$ das hiperplasias com atipias progridem para câncer em um intervalo de 13 anos.

Em muitos casos o carcinoma apresenta-se focal, bem diferenciado e superficial. Há hipóteses que seu desenvolvimento ocorreria a partir de um pólipo ou de regiões do endométrio que não respondem à ação da progesterona. O processo de transformação neoplásica no endométrio envolve várias etapas, com múltiplas possibilidades de seqüências genotípicas, resultando em aspectos tumorais diversos ${ }^{7,9}$.

Por muitos anos, a dilatação e curetagem foram métodos de escolha para o diagnóstico de carcinoma de endométrio. No entanto, Stock e Kanbour ${ }^{10}$ evidenciaram que em $60 \%$ das curetagens realizadas, menos da metade da cavidade uterina era curetada, questionando a acurácia diagnóstica deste método. Mais recentemente, alguns trabalhos confirmaram as limitações da curetagem, evidenciando índices de falso-negativo de até $10 \%{ }^{11}$. Segundo Guelmann e
Rotstein ${ }^{12}$, 20\% das pacientes submetidas a histerectomia que apresentavam canal endocervical comprometido tinham curetagem negativa, e em $100 \%$ dos casos que demonstravam envolvimento cervical à curetagem fracionada, não havia invasão na peça cirúrgica.

O método diagnóstico ideal otimiza a sensibilidade, a especificidade e a eficácia diante dos custos, ao mesmo tempo em que maximiza o conforto da paciente e uma clara compreensão do seu estado $^{13}$. Desta forma, a histeroscopia associada à biópsia resulta em economia de tempo para o ginecologista e de custos e do risco anestésico para a paciente, obtendo-se maior sensibilidade e especificidade que o ultra-som e a curetagem nas patologias da cavidade uterina ${ }^{14-16}$.

O diagnóstico histeroscópico das hiperplasias endometriais é norteado pela classificação proposta pela Sociedade Internacional dos Patologistas Ginecologistas, que é baseada em critérios de arquitetura glandular, dividindo as hiperplasias em simples e complexas, bem como em critérios celulares, de acordo com a presença de atipias ${ }^{17}$.

As hiperplasias simples, na sua fase inicial, têm população glandular aumentada, diminuindo sobremaneira os espaços interglandulares, adquirindo um aspecto ondulado, róseo e sem vascularização. Pode ser confundido com o endométrio proliferativo, mas é mais denso, compacto, avascular e mais hemorrágico ao contato. As hiperplasias com mais tempo de evolução têm aspecto polipóide e podem ser confundidas com o endométrio secretor, branco e avascular e às vezes de difícil diferenciação. Esse aspecto histeroscópico é chamado de hiperplasia de baixo risco ${ }^{17}$.

As hiperplasias complexas inicialmente apresentam um endométrio secretor espesso, mas com vascularização hipertrófica e vasos de trajeto curto. Pode haver áreas de necrose com formação de vesículas ou cistos, no alto das formações polipóides. Quanto maior é o tempo de evolução da doença, com mais freqüência podemos encontrar aderências mucosas. Em um estágio mais avançado, temos a formação de úlceras, cistos e abundante vascularização superficial. A esse aspecto histeroscópico, chamamos de hiperplasia de alto risco ${ }^{17}$.

O adenocarcinoma apresenta grandes alterações da arquitetura do endométrio, com intensa irregularidade vascular, vasos anômalos com aparência arborescente circundando grupos de orificios glandulares e áreas de necrose freqüentes, muitas vezes se confundindo com o aspecto histeroscópico da hiperplasia atípica ou adenomatosa ${ }^{17}$.

A histeroscopia também possibilita a avali- 
ação da extensão das lesões endometriais por hiperplasia e lesões precursoras do adenocarcinoma, do acometimento do canal endocervical e proporciona a possibilidade de biópsia dirigida ou orientada do endométrio ${ }^{15}$. Há alguns anos havia controvérsias quanto à contra-indicação da realização de histeroscopia na suspeita de adenocarcinoma, devido à possível disseminação de células neoplásicas na cavidade endometrial. Em estudo recente, ficou comprovado que não há alteração no prognóstico da doença, sendo hoje utilizada a histeroscopia como um dos métodos de avaliação da extensão da lesão e do comprometimento do canal endocervical, tendo em vista a importância do estadiamento histeroscópico para orientar o tratamento mais adequado ${ }^{17,18}$.

No adenocarcinoma de endométrio inicial, Marabini ${ }^{19}$ estabeleceu um estadiamento por meio da imagem histeroscópica de acordo com a extensão do acometimento na cavidade uterina. O estádio alfa ocorre quando o tumor atinge até $1 / 3$ da cavidade uterina, estádio beta quando acomete até $2 / 3$ da cavidade e o estádio gama quando toda a extensão da cavidade uterina está comprometida.

A Federação Internacional de Ginecologia e Obstetrícia determinou graduações tumorais segundo padrões de arquitetura celular à histologia, com o objetivo de estabelecer prognóstico e esquemas de tratamento para o câncer de endométrio. Os tumores são chamados de grau 1 (G1) quando 95\% do tumor forma glândulas, grau 2 (G2) quando exibe 6 a $50 \%$ de crescimento sólido e grau 3 (G3) quando mais de 50\% do tumor apresenta padrão sólido 9 .

\section{Pacientes e Métodos}

Os dados foram coletados de forma retrospectiva por meio de questionário padronizado respondido por cinco centros de histeroscopia brasileiros, localizados em São Paulo, Rio de Janeiro, Salvador, Caxias do Sul e Porto Alegre. Foram analisados 6.466 procedimentos histeroscópicos no período de maio de 1993 a agosto de 1997. No questionário constava o número de histeroscopias realizadas, os casos de adenocarcinoma diagnosticados por histeroscopia, os casos de adenocarcinoma confirmados pela histologia e número de pacientes na pré e pós-menopausa. Também se analisou o estadiamento histeroscópico do adenocarcinoma de endométrio entre os casos no estadio I, conforme a extensão do acometimento da cavidade uterina.

Por se tratar de centros que tiveram a mesma orientação quanto à técnica, foi possivel ade- quada padronização da técnica de exame histeroscópico. Todos os exames foram realizados em regime ambulatorial sem anestesia, utilizando-se $\mathrm{o} \mathrm{CO}_{2}$ como meio de distensão da cavidade uterina com pressão em torno de $100 \mathrm{mmHg}$ e fluxo de gás de até $75 \mathrm{~mL} / \mathrm{min}$.

Para o diagnóstico histeroscópico de adenocarcinoma, foram consideradas as alterações de arquitetura endometrial, freqüentemente com formações polipóides presentes e vascularização anômala com aparência arborescente, circundando grupos de orifícios glandulares. O diagnóstico endoscópico foi confirmado com a obtenção de amostras de endométrio para estudo histológico.

Foi realizada biópsia orientada sempre que se encontrou imagem de desarranjo da arquitetura do endométrio. As biópsias foram orientadas pelo histeroscópio e realizadas com a utilização de cureta de Novak. A análise histológica do material obtido com as biopsias foi realizada de forma descentralizada, nas cidades onde os questionários foram respondidos.

As pacientes portadoras de carcinoma inicial foram classificadas segundo Marabini, conforme a extensão da cavidade uterina acometida, em estádios alfa, beta e gama.

Os graus de diferenciação histológica segundo a Federação Internacional de Ginecologia e Obstetrícia também foram avaliados, classificando-os em graus G1, G2 e G3, conforme padrão de crescimento sólido não escamoso ou não morular.

A presente pesquisa foi realizada obedecendo às normas e diretrizes éticas da Resolução 196/ 96 do Conselho Nacional de Saúde do Ministério da Saúde, em que é garantido o caráter confidencial da identidade das pacientes e das informações obtidas neste estudo.

\section{Resultados}

O diagnóstico histeroscópico de adenocarcinoma de endométrio foi realizado em 92 casos $(1,4 \%)$, sendo confirmado pela histologia em 79 $(1,2 \%)$, em um total de 6.466 pacientes examinadas. Não houve casos nos questionários avaliados de adenocarcinoma de endométrio não diagnosticados pela histeroscopia, cujo resultado histológico apresentasse positivo para esta doença.

Para o diagnóstico histeroscópico de adenocarcinoma de endométrio, confirmado por exame histológico, obtivemos sensibilidade de $85,9 \%$, especificidade de $100 \%$, valor preditivo positivo de $100 \%$ e valor preditivo negativo de $98,6 \%$.

Em relação ao perfil reprodutivo, o questionário levou em conta dois grupos distintos de pa- 
cientes, um na pré e outro na pós-menopausa. As pacientes da pré-menopausa somaram 3.845 casos, entre as quais o diagnóstico histeroscópico de carcinoma de endométrio foi obtido em $9(0,2 \%)$ casos e confirmado pela histologia em $8(0,2 \%)$. $\mathrm{Na}$ pós-menopausa foram examinadas $2.621 \mathrm{pa}-$ cientes, tendo sido diagnosticado por histeroscopia adenocarcinoma de endométrio em 83 (3,2\%) casos, com $71(2,7 \%)$ casos confirmados pela histologia (Figura 1).

$\square$ Diagnóstico histeroscópico $\square$ Confirmação histológica

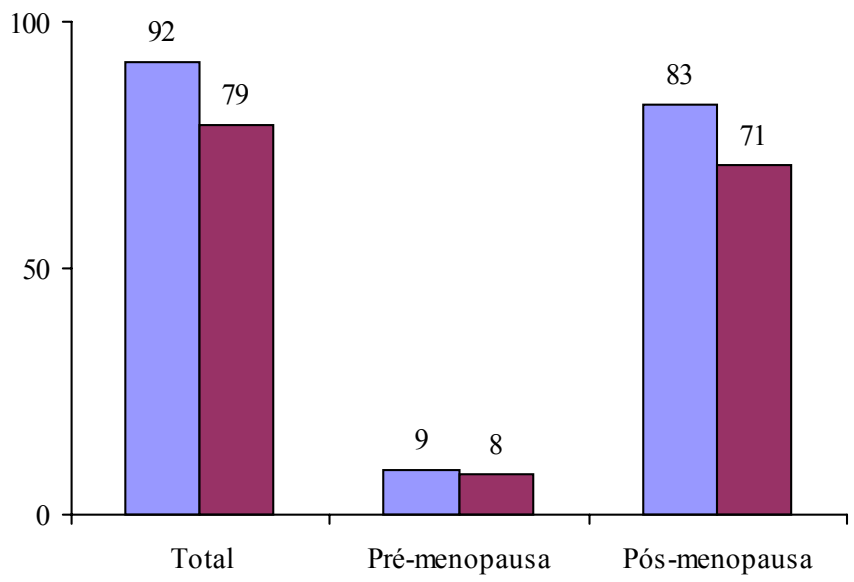

Figura 1 - Diagnóstico histeroscópico e confirmação histológica do adenocarcinoma do endométrio.

Em relação ao estadiamento histeroscópico do adenocarcinoma, em 26 casos diagnosticados por histeroscopia e confirmados pela histologia, 12 acometiam somente um terço da cavidade uterina, constituindo o estádio alfa, 6 foram considerados estádio beta e 8 acometiam toda cavidade uterina, determinados como estádio gama (Figura 2). Desses 26 casos analisados, 22 eram de tipo histológico G1, 2 de tipo G2 e 3 casos eram G3.

$\square$ Casos de adenocarcinoma estadiados pela histeroscopia

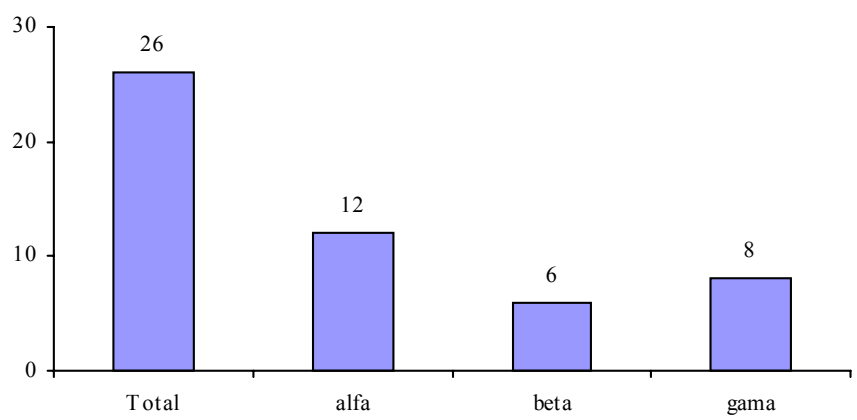

Figura 2 - Estadiamento histeroscópico dos casos de adenocarcinoma.

\section{Discussão}

A realização do procedimento histeroscópico não apresenta complicações, quando é utilizada técnica adequada. Em algumas pacientes na pósmenopausa, que representam o grupo com maior incidência desta neoplasia, pode haver dificuldades na passagem do aparelho através do canal endocervical. Este inconveniente tem diminuído em função da progressiva utilização de equipamento óptico de menor calibre.

Os sintomas do adenocarcinoma de endométrio freqüentemente são inespecíficos. Em $80 \%$ das pacientes ocorre sangramento uterino anormal e em $10 \%$ dos casos há leucorréia ${ }^{20}$.

$\mathrm{O}$ aspecto macroscópico histeroscópico permite um diagnóstico confiável e, se associado à biópsia orientada ou dirigida, pode-se obter acuidade em torno de $92 \%$ nos casos de hiperplasias endometriais atipicas e adenocarcinoma ${ }^{17}$. No entanto, em questionário semelhante enviado a 115 centros europeus de oncologia ginecológica sobre a abordagem no adenocarcinoma de endométrio, apesar da grande difusão da histeroscopia na Europa, este procedimento era realizado em menos da metade destes serviços ${ }^{21}$.

Analisando 2.074 histeroscopias com indicação de sangramento uterino anormal no menacme, Marabini ${ }^{19}$ encontrou adenocarcinoma de endométrio em $0,3 \%$ dos casos e hiperplasia endometrial em 9,7\%. O mesmo autor, quando analisou 1.475 histeroscopias com a mesma indicação na pós-menopausa, observou a ocorrência de adenocarcinoma em $6,2 \%$ e hiperplasia endometrial em $6,1 \%$ dos casos. Tantini e Mencaglia, ${ }^{21}$ realizaram 2.700 histeroscopias em pacientes menopausadas com sangramento uterino anormal e encontraram $8 \%$ de adenocarcinomas, com $97 \%$ de confiança no diagnóstico histeroscópico dessa doença.

Em estudo de Motashaw e Dave ${ }^{22}$ com 370 pacientes que apresentavam sangramento uterino anormal, a histeroscopia revelou adenocarcinoma em $1,3 \%$ dos casos. Dones e colaboradores ${ }^{23}$ realizaram 215 histeroscopias em pacientes com sangramento uterino pós-menopausa, encontrando 4 a $5 \%$ destas hiperplasias de alto risco e adenocarcinomas.

Folegatti e colaboradores ${ }^{24}$ avaliaram 3.020 pacientes com sangramento uterino anormal por meio de histeroscopia e biópsia e encontraram 84 casos de adenocarcinoma, sendo que em 80 a histeroscopia diagnosticou corretamente. Em 4 casos de falso-negativo a histeroscopia diagnosticou hiperplasia de baixo risco ou pólipos. Por outro lado, outros 4 casos foram diagnosticados como 
suspeitos de adenocarcinoma endometrial pela histeroscopia, mas a histologia se mostrou negativa (falso-positivo). A sensibilidade e especificidade dos resultados e sua taxa de acurácia diagnóstica para adenocarcinoma endometrial foram de 95,2\%, 99,9\% e 99,8\%, respectivamente.

Valli e Zuppi ${ }^{25}$ analisaram 242 histeroscopias, encontrando sensibilidade para hiperplasia complexa com atipia e adenocarcinoma de $94,1 \%$ e especificidade de $94 \%$. Valores superiores encontrados na literatura em relação à sensibilidade e especificidade obtidos neste estudo provavelmente ocorreram devido ao caráter multicêntrico dos exames histeroscópicos, bem como à descentralização da leitura dos exames histológicos.

Em relação ao estadiamento histeroscópico dos casos de adenocarcinoma, o número de casos analisados foi menor que o número total em função de perda de acompanhamento dos casos. Os cinco centros de histeroscopia participantes deste estudo colaborativo são centros de referência; por muitas vezes a paciente é operada por seu ginecologista particular, não dispondo de alguns resultados do estadiamento anátomo-cirúrgico, que foi realizado de forma descentralizada. A definição da topografia tumoral no estádio I tem importância como fator prognóstico. Quando se comparou o estadiamento histeroscópico com a avaliação histológica pós-histerectomia, Marabini ${ }^{19}$ encontrou tumor menor ou igual a $2 \mathrm{~cm}$ no estádio alfa em $83 \%$ dos casos.

Concluímos que a histeroscopia tem um papel importante não só no diagnóstico de lesões precursoras, mas também para orientar tratamentos mais adequados no câncer de endométrio. No diagnóstico de tumores em estádio I-alfa, a histeroscopia pode orientar tratamentos menos agressivos. Por outro lado, na vigência de invasão do canal cervical (estádio II), a histeroscopia orienta tratamentos mais agressivos. Nos casos de diagnóstico de tumores difusos por toda a cavidade uterina (estádio gama), a histeroscopia pode indicar maior risco de invasão miometrial/ metastatização. Assim, o papel da histeroscopia no manejo do adenocarcinoma de endométrio é indiscutivel. A obtenção de mais dados epidemiológicos a partir de questionários nacionais multicêntricos certamente deve conduzir à criação de programas de prevenção e diagnóstico precoce do câncer de endométrio.

\section{ABSTRACT}

Purpose: to perform a census about the frequency of endometrial adenocarcinoma of women submitted to diagnostic hysteroscopy in five Brazilian hysteroscopic centers in São Paulo, Rio de Janeiro, Salvador, Caxias do Sul and Porto Alegre.

Methods: information was collected from standard questionnaires about the presence of endometrial adenocarcinoma, hysteroscopic staging and histologic type, in pre- and postmenopausal women.

Results: among 6,466 hysteroscopic procedures, endometrial adenocarcinoma was present in 92 patients (1.4\%), confirmed by histology in 79 (1.2\%) cases. For the hysteroscopic diagnosis of endometrial adenocarcinoma confirmed by histology, a sensitivity of $85.9 \%$, specificity of $100 \%$, positive predictive value of $100 \%$ and negative predictive value of $98.6 \%$ were obtained. In the premenopausal group, among 3,845 hysteroscopic exams, endometrial cancer was present in $83(3.2 \%)$ and confirmed by histology in 71 cases (2.7\%).

Conclusions: this study points out the importance of epidemiological methods in the diagnostic and prevention programs of endometrial cancer, specially in postmenopause, revealing the need for further epidemiological studies on endometrial adenocarcinoma diagnostis and prevention programs.

KEY WORDS: Hysteroscopy. Endometrial neoplasms. Cancer screening. Endometrial neoplasms, epidemiology. Endometrial neoplasms, histology.

\section{Referências}

1. Rose PG. Endometrial carcinoma. N Engl J Med 1996; 335:640-9.

2. Silverberg E, Lubera JA. Cancer statistics. CA Cancer J Clin 1988; 38:5-22.

3. Ligniéres B, Moyer DL. Influence of sex hormones on hyperplasia/carcinoma risks. In: Lobo RA, editor. Treatment of the Postmenopausal Woman: basic and clinical aspects. 1st ed. New York: Raven Press; 1994. p.373-9.

4. Brasil. Ministério da Saúde. Secretaria Nacional de Assistência à Saúde. Instituto Nacional do Câncer. Coordenação de Programas de Controle de Câncer - Pró-Onco. Estimativa da incidência e mortalidade por câncer no Brasil 1998. Rio de Janeiro: Pro-Onco/INCA; 1998. p.18.

5. Gordon J, Reagan JW, Finkle WD, Ziel HK. Estrogen and endometrial carcinoma. An independent pathology review supporting original risk estimate. N Engl J Med 1977; 297:570-1.

6. Ziel HK. Estrogen's role in endometrial cancer. Obstet Gynecol 1982, 60:509-15.

7. Creasy GW, Kafrissen ME, Upmalis D. Review of the endometrial effects of estrogens and progestins. Obstet Gynecol Surv 1992; 47:654-78. 
8. Mazur MT, Kurman RJ. Endometrial carcinoma. In: Mazur MT, Kurman RJ, editors. Diagnosis of Endometrial Biopsies and Curettings. A practical approach. 1st ed. New York: Springer-Verlag; 1995. p.184-218.

9. Kurman RJ, Kaminski PF, Norris HJ. The behavior of endometrial hyperplasia. A long-term study of untreated hyperplasia in 170 patients. Cancer 1985; 56:403-12.

10.Stock RJ, Kanbour A. Prehysterectomy curettage. Obstet Gynecol 1975; 45:537-41.

11.Stovall TG, Solomon SK, Ling FW. Endometrial sampling prior to hysterectomy. Obstet Gynecol 1989; 73:405-9.

12.Guelmann S, Rotstein S. Curetagem semiótica fracionada: avaliação de sua fidelidade diagnóstica no carcinoma do endométrio. Rev Bras Ginecol Obstet 1994; 16:55-8.

13. Cooper JM, Brady RM. Hysteroscopy in the management of abnormal uterine bleeding. Obstet Gynecol Clin North Am 1999; 26:217-36.

14.Gimpelson RJ. Panoramic hysteroscopy with directed biopsies vs dilatation and curettage for accurate diagnosis. J Reprod Med 1984; 29:575-8.

15.Gimpelson RJ, Rappold HO. A comparative study between panoramic hysteroscopy with directed biopsies and dilatation and curettage. A review of 276 cases. Am J Obstet Gynecol 1988; 158:489-92.

16.Brill AI. What is the role of hysteroscopy in the management of abnormal uterine bleeding? Clin Obstet Gynecol 1995; 38:319-45.
17. Mencaglia L. Hysteroscopy and adenocarcinoma. Obstet Gynecol Clin North Am 1995; 22:573-9.

18.Donnez J. Hysteroscopy in the diagnosis of specific disorders. In: Donnez J, editor. Laser Operative Laparoscopy and Hysteroscopy. 1st ed. Leuven: Nauwelaerts; 1989. p.223.

19.Marabini A. The state of the art on endometrial cancer. Gynaecol Endosc 1995; 4:6-9.

20.Platz CE, Benda JA. Female genital tract cancer. Cancer 1995; 75 Suppl:270-94.

21.Tantini C, Mencaglia L. Endometrial cancer: endoscopic diagnosis and treatment. Gynaecol Endosc 1994; 3:22-9.

22.Motashaw ND, Dave S. Diagnostic and therapeutic hysteroscopy in the management of abnormal uterine bleeding. J Reprod Med 1990; 35:616-20.

23.Dones F, Maniscalco V, Cadili G. L'isteroscopia come metodica diagnostica nelle metrorragie della postmenopausa. Minerva Ginecol 1992; 44:433-5.

24.Folegatti MR, De Natale S, Levato F, Martinello R, Grandi E, Mollica G. The hysteroscopy in the diagnosis of endometrial adenocarcinoma. Gynaecol Endosc 1994; 3:9-13.

25.Valli E, Zupi E. A new hysteroscopic classification of and nomenclature for endometrial lesions. J Am Assoc Gynecol Laparosc 1995; 2:279-83.

Recebido em: 31/7/2001 Aceito com modificações em: 6/12/2001

\title{
COMUNICADO AOS ASSOCIADOS E LEITORES
}

$$
\begin{gathered}
\text { FEBRASGO na } \\
\text { Internet }
\end{gathered}
$$

Para informações e consultas temos à disposição quatro endereços eletrônicos:

\author{
febrasgopresiden@uol.com.br \\ secretaria_executiva@febrasgo.org.br \\ publicacoes@febrasgo.org.br \\ tego_habilitacoes@febrasgo.org.br
}

